\title{
Diphtheria or Streptococcal Pharyngitis: A Case Report Highlighting the Diagnostic Dilemma in the Post-vaccination Era
}

\author{
Venkataramana Kandi $^{1}$, Ritu Vaish ${ }^{2}$ \\ 1. Clinical Microbiology, Prathima Institute of Medical Sciences, Karimnagar, IND 2. Microbiology, Prathima Institute \\ of Medical Sciences, Karimnagar, IND
}

Corresponding author: Venkataramana Kandi, ramana20021@gmail.com

\begin{abstract}
Diphtheria is an acute, highly infectious, toxigenic, and vaccine-preventable disease that commonly affects children under 12 years of age. The incidences of diphtheria have significantly dropped due to vaccination with diphtheria, pertussis, tetani (DPT). Recently, there is an increasing trend in reports of diphtheria throughout the world and specifically from developing countries. According to a World Health Organization (WHO) report, more than $80 \%$ of the global diphtheria cases in the post-vaccination era were from India and Indonesia. This could probably be signaling its re-emergence, which may be attributed to several factors that include incomplete immunization. Pharyngitis caused by group A Streptococcus is most frequently seen in children and can be clinically similar in presentation to diphtheria. We share our experience of managing a case of an eight-year-old child, who was clinically suspected to be suffering from diphtheria.
\end{abstract}

Categories: Pediatrics, Infectious Disease, Epidemiology/Public Health

Keywords: diphtheria, tonsillitis, vaccine preventable disease, corynebacterium diphtheriae, children

\section{Introduction}

Diphtheria is a bacterial infection caused by Corynebacterium diphtheriae (C. diphtheriae). It is transmitted among humans through the respiratory route (aerosols). Diphtheria is an acute, severely debilitating illness, usually affecting children less than 12 years of age. In diphtheria, there is a formation of thick pseudomembrane or a leathery sheet (diphtheros) on the posterior pharyngeal wall, formed by the accumulation of bacterial cells, epithelial cells, and other inflammatory cells. This causes a mechanical obstruction and results in difficulty in swallowing and, in some cases, dyspnoea (difficulty in breathing). Since C. diphtheriae is a toxin-producing bacteria, the exo-toxin is released by the bacteria, which then enters the blood/general circulation, resulting in several, other complications in the infected patients. Clinically, diphtheria may present as faucial, laryngeal, cutaneous, and others. C. diphtheriae has been noted to occur in three different strains/types depending on the intensity of the infection they cause, the gravis type produces a severe infection, the intermedius type results in moderate infection, and the mitis strain causes a mild type of diphtheria [1].

Received 11/13/2019

$11 / 15 / 2019$ Review ended 11/17/2019 Published 11/18/2019

(c) Copyright 2019 Kandi et al. This is an open access article distributed under the terms of the Creative Commons Attribution License CC-BY 3.0., which permits unrestricted use, distribution, and reproduction in any medium, provided the original author and source are credited.
Clinical and laboratory diagnosis assumes great significance to efficiently manage the suspected cases of diphtheria and minimize the resultant morbidity and mortality. Patients with diphtheria usually present with sore throat and fever, which also is the presentation of patients suffering from infection with the more common bacteria, Streptococcus pyogenes (beta-hemolytic streptococci/group A streptococci) and other microbial infections [2]. In view of the fact that diphtheria, pertussis, and tetani (DPT) has been in regular use as a vaccine against diphtheria for many years, the clinical cases of diphtheria have almost been negligible. Most pediatricians are now in a dilemma regarding the prevalence of diphtheria and probably misdiagnose the potential cases of diphtheria as a streptococcal sore throat. This type of diagnosis and a delay in the appropriate management of cases of diphtheria may result in severe complications among infected patients and could result in mortality.

Recently, there have been some reports of the re-emergence of diphtheria, which should be considered as a cause of serious concern [3-5]. We report our experience of managing a clinically diagnosed case of diphtheria and emphasize its significance in the era of vaccination.

\section{Case Presentation}

An eight-year-old boy was brought to the casualty department attached to the Prathima Institute of Medical Sciences with the chief complaints of fever, malaise, vomiting, and difficulty in swallowing. The boy was admitted to the pediatric intensive care unit (PICU) for further evaluation. The boy's parents complained of acute onset of low-grade fever three days back. The boy was previously normal and was going to school regularly. The fever episodes were not associated with any type of skin rash. Three to four episodes of vomiting per day were noted along with the fever. The vomiting was non-projectile, non-bilious, blood- 


\section{Cureus}

tinged, and was stimulated by both solid and liquid food intake. The boy also complained of pain in the throat and had difficulty swallowing. The patient had a loss of appetite, gave a history of high-colored urine, and had generalized weakness.

No previous history of similar complaints in the patient, as well as his two other siblings, was reported. There was no documented evidence/medical record that the patient was immunized with DPT although the parents claimed that the patient was immunized according to the national immunization schedule.

On clinical examination, the patient's vitals were all good. A noisy breath, probably due to the infection in the throat, was noted, without any dyspnoea. Clinical examination of the pharynx showed grade IV tonsillitis with a grayish-white membranous patch covering the tonsil, which was extending towards the soft palate. The posterior pharyngeal wall revealed congestion, with both sides of the tonsil showing enlargement. The uvula was central, oedematous, showed congestion, and was bleeding on touch.

General physical examination of the patient revealed sunken eyes, loss of the buccal pad of fat, a prominent maxilla, and a scaphoid abdomen. The patient was noted to be underweight $(20 \mathrm{~kg})$ as against the recommended weight at the same age ( $32 \mathrm{~kg}$ ) and was $133 \mathrm{~cm}$ tall as against the recommended height (140 $\mathrm{cm})$ at a corresponding age.

The patient's parents reported a low-calorie intake of $1200 \mathrm{KCal} /$ day as against the recommended 1920 $\mathrm{KCal} /$ day. Also, the patient was only taking $24 \mathrm{~g}$ of protein against the daily recommended intake of 38.4 $\mathrm{g} /$ day. Considering this, the patient was diagnosed/categorized as suffering from protein-energy malnutrition.

The complete blood picture showed neutrophilic leucocytosis, and borderline platelet counts (1.5 lakh cells $\left./ \mathrm{mm}^{3}\right)$. The patient had raised C-reactive protein (CRP) $(2.4 \mathrm{mg} / \mathrm{dL})$ and erythrocyte sedimentation rate (ESR) $(40 \mathrm{~mm})$.

A preliminary diagnosis of grade IV tonsillitis was made, and a throat swab was sent to the clinical microbiology laboratory for a direct Gram's stain, culture, and sensitivity. On direct Gram's stain of the throat swab, plenty of Gram-positive bacilli were observed, with occasional Gram-positive cocci, as shown in Figure 1.

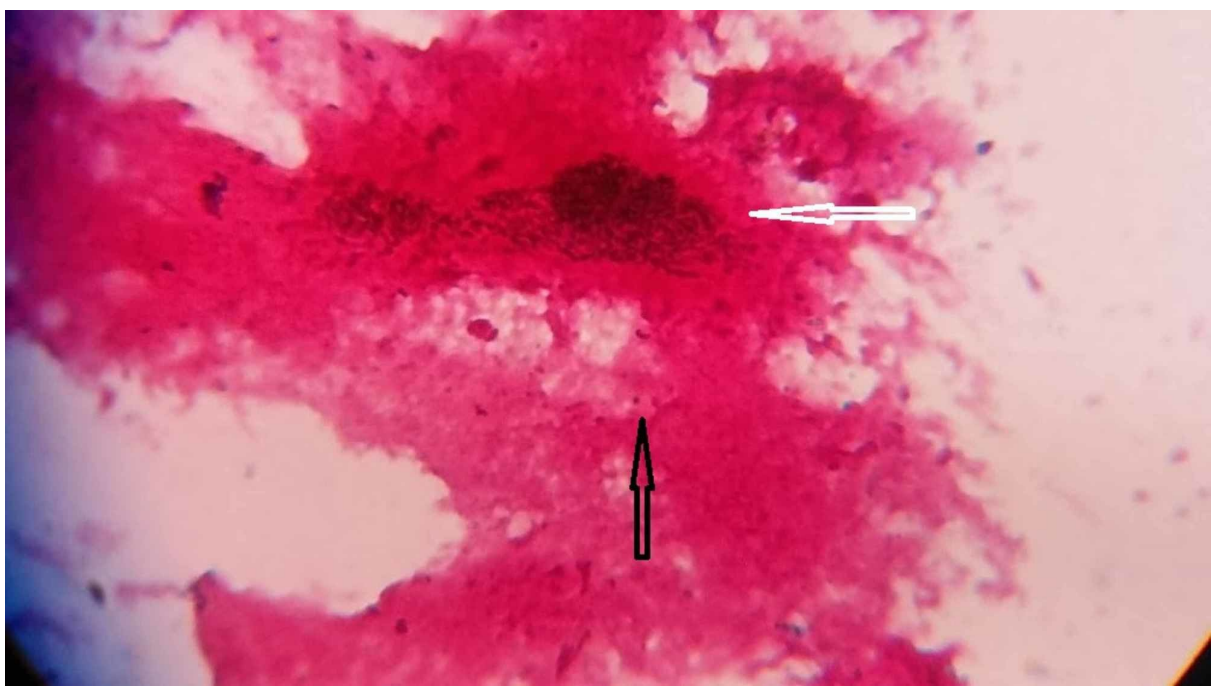

FIGURE 1: Direct Gram's stain of the throat swab showing plenty of Gram-positive bacilli (white arrow) with occasional Gram-positive cocci (black arrow)

Culture on blood agar showed 2-3 millimeter, small, round, white-cream-colored non-hemolytic colonies along with 1-2-millimeter, pinpoint, translucent beta-hemolytic colonies, as shown in Figure 2. 


\section{Cureus}

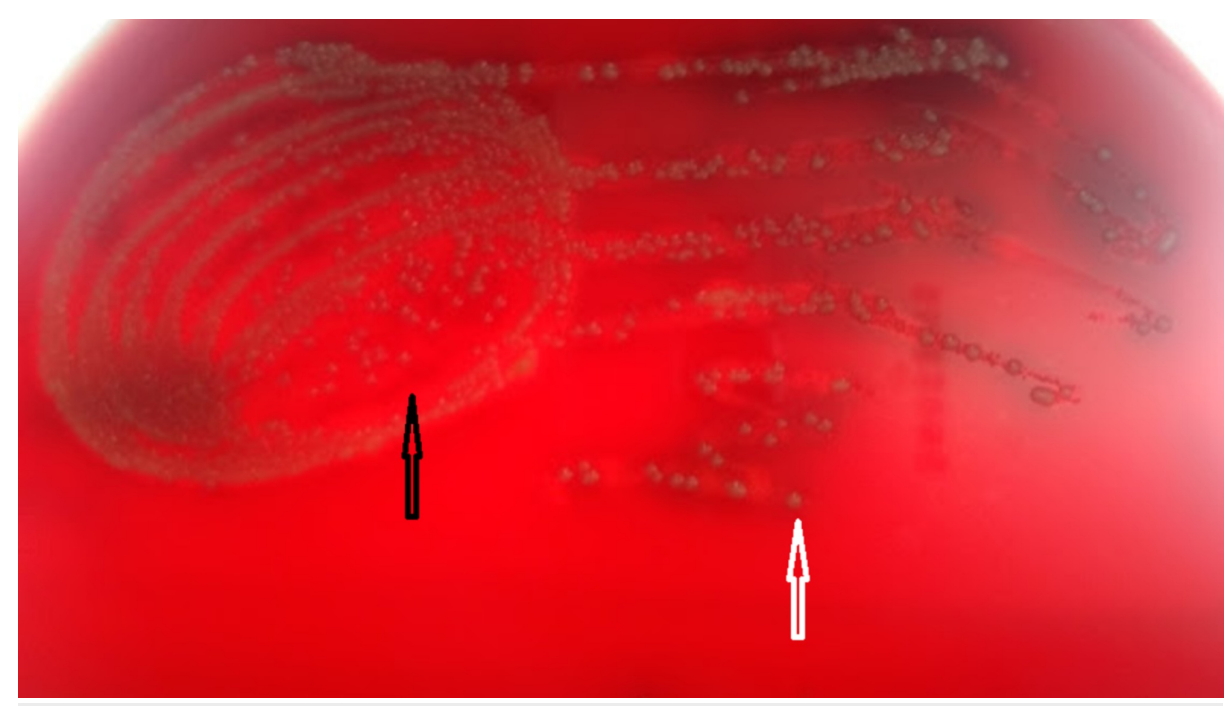

FIGURE 2: Culture on blood agar showing opaque, non-hemolytic colonies (white arrow) and translucent beta-hemolytic colonies (black arrow)

Gram's stain of the small and the pinpoint colonies revealed gram-positive bacilli and gram-positive cocci in pairs respectively as shown in Figure 3.

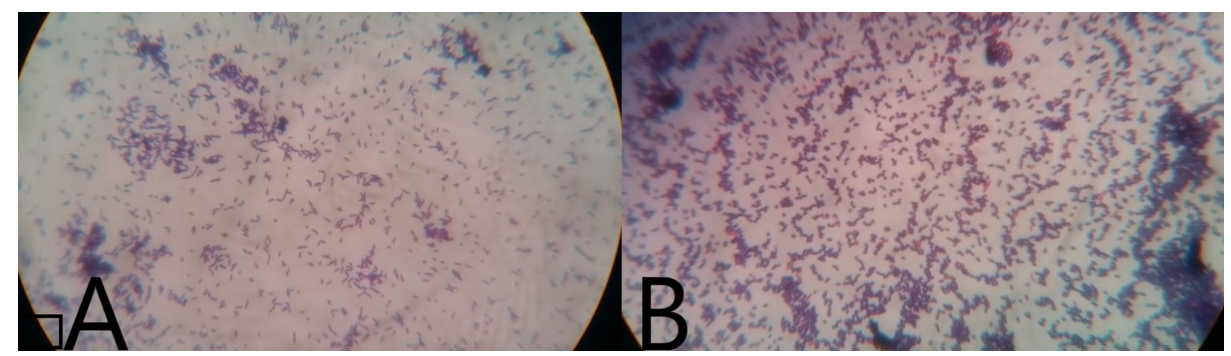

FIGURE 3: Gram's stain of the small colonies showing gram-positive bacilli $(A)$ and the pinpoint colonies showing gram-positive cocci $(B)$

The colonies on blood agar were non-hemolytic (C. diphtheriae forms hemolytic colonies), glossy (gravis type is matt-like) in appearance, raised (intermedius are flat), and were glistening and butyrous (butter-like) in texture, as shown in Figure 4.

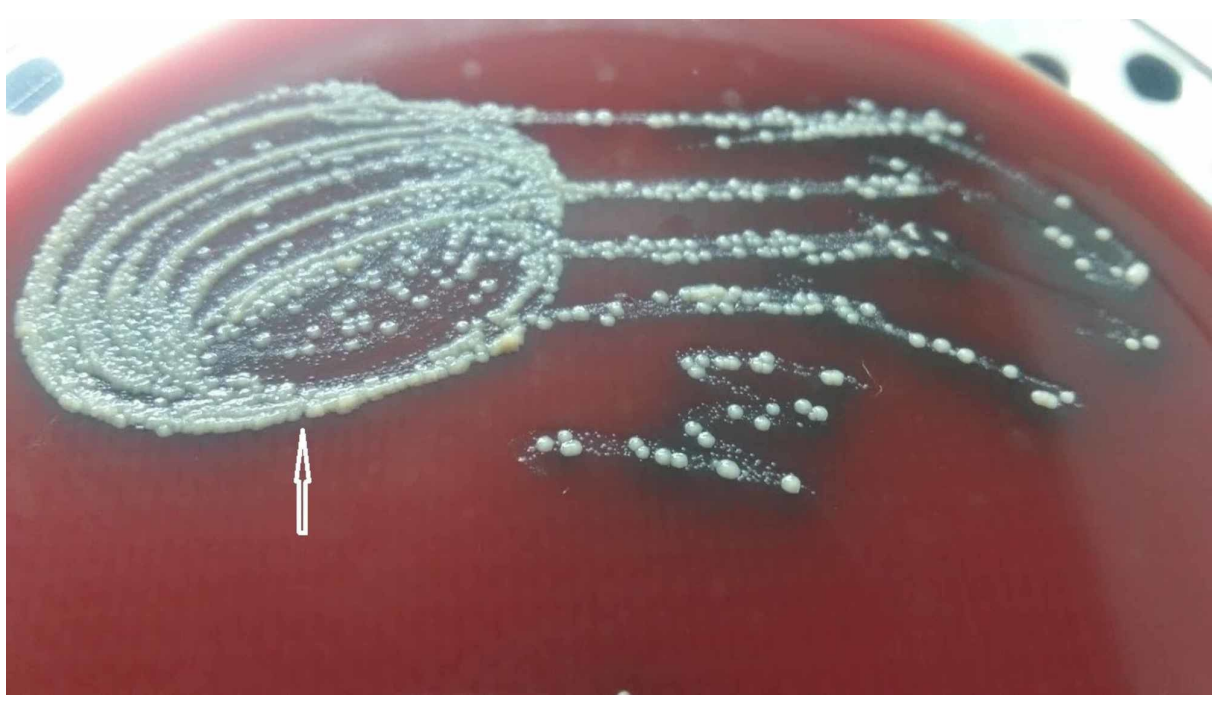




\section{Cureus}

FIGURE 4: White to cream-colored, raised, glossy, and butyrous colonies (white arrow) of diphtheria like bacteria on blood agar

They were catalase-positive, non-motile, and non-fermenters on triple sugar iron agar (TSI). The Grampositive bacilli were identified as morphologically and biochemically resembling C. diphtheriae. The grampositive cocci were catalase-negative and were identified as Streptococcus species (Streptococcus Spp).

Both the gram-positive cocci and the bacilli showed varied sensitivity patterns using the Kirby-Bauer disk diffusion method. The antimicrobial susceptibility pattern of gram-positive bacilli showed sensitivity to vancomycin, linezolid, tetracycline, and ofloxacin. Resistance was observed against penicillin, oxacillin, clindamycin, ciprofloxacin, cefotaxime, cefepime, cefoperazone, ceftriaxone, ceftazidime, amikacin, gentamicin, and piperacillin-tazobactam, as shown in Figure 5.

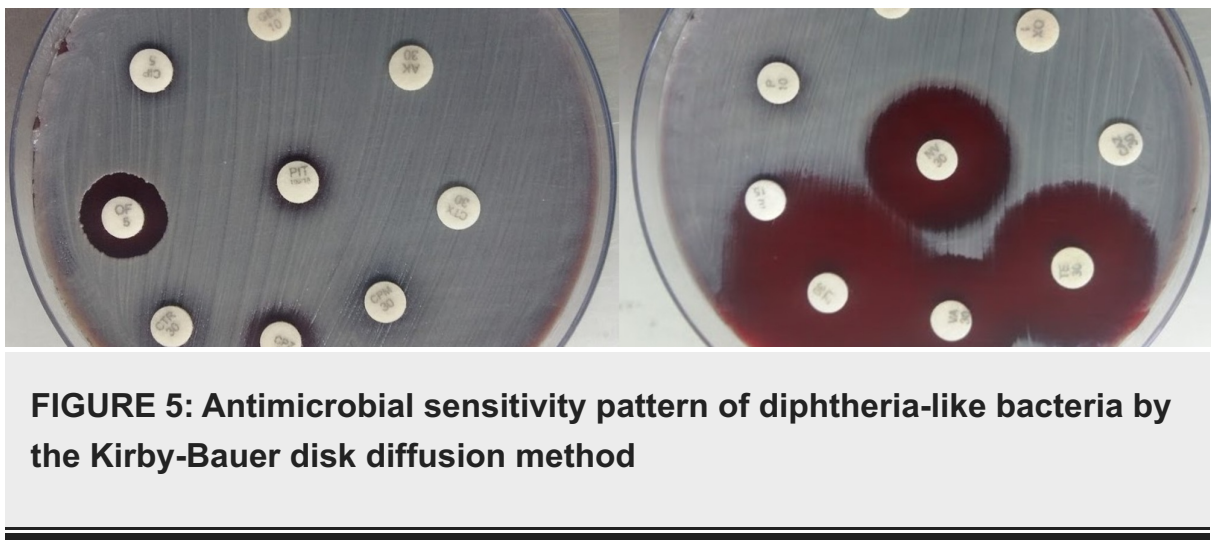

The Streptococcus spp. isolated was sensitive to amikacin, gentamicin, ciprofloxacin, ofloxacin, trimethoprim-sulfamethoxazole, vancomycin, linezolid, tetracycline, and piperacillin-tazobactam. Resistance was noted against penicillin, erythromycin, clindamycin, oxacillin, cefotaxime, cefepime, cefoperazone, ceftriaxone, and ceftazidime, as shown in Figure 6.

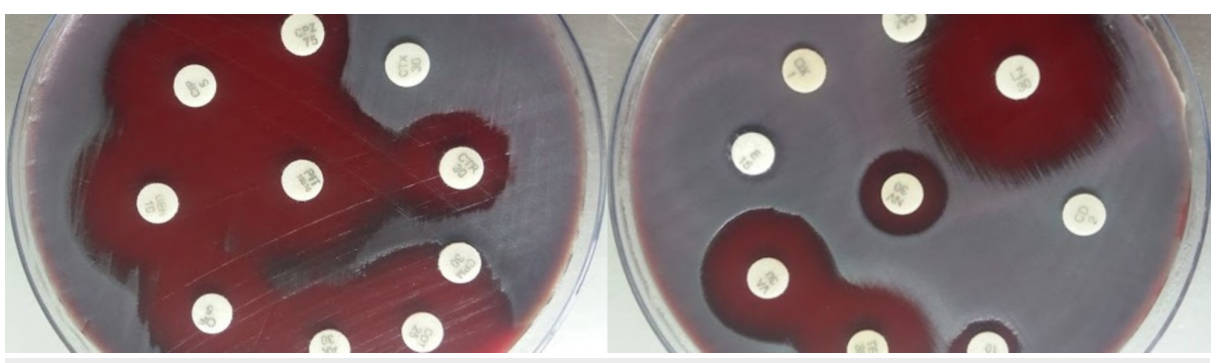

FIGURE 6: Antimicrobial sensitivity pattern of Streptococcus species by the Kirby-Bauer disk diffusion method

Considering the transmissibility of the infection, the patient was put under isolation. Treatment was initiated with 80,000 units of diphtheria antitoxin through the intravenous route. Since the isolated organisms were resistant both to penicillin and erythromycin, the drugs of choice in the case of suspected diphtheria, and because the patient also had beta-hemolytic Streptococci, the patient was started on a course of piperacillin-tazobactam and amikacin. The patient had a gradual and uneventful recovery.

The bacterium morphologically resembling $C$. diphtheriae was not confirmed using the standard anti-toxin by the Elek's gel precipitation test due to the unavailability of a suitable identification system. Also, the close household contacts were neither screened nor administered prophylactic antibiotics as suggested/recommended by the World Health Organization (WHO) because of the non-cooperation of the patient's parents.

\section{Discussion}

Diphtheria is a highly infectious and reportable bacterial disease that is prevalent throughout the world. The introduction and success of the DPT vaccination had been instrumental in the control of the disease, which 
mostly affects children below 12 years of age, causing significant morbidity and mortality. Streptococcal sore throat, caused by group A Streptococci is another upper respiratory tract infection prevalent among children. The clinical presentation of both diphtheria and streptococcal pharyngitis appears similar, and the clinical diagnosis becomes difficult. Assuming that the cases of diphtheria are almost negligible due to DPT vaccine, and with limited knowledge of the prevalence of diphtheria in the post-vaccination era, most physicians/pediatricians may misdiagnose the diphtheria cases as streptococcal infections. Such diagnoses may result in the spread of diphtheria among the contacts and also delay the initiation of treatment.

\section{Global scenario of diphtheria}

The re-emergence of diphtheria has been a point of discussion almost since the past decade. The occurrence of diphtheria in the post-vaccination era was attributed to the discrepancies (incomplete vaccination) in the immunization. Most infections in the post-vaccination era have been noted to emerge from the developing nations, which include the incidences of outbreaks from Indonesia, Bangladesh, and Yemen [6-9].

Isolated reports of outbreaks of diphtheria have also been reported from the developed nations, including the United States of America (USA). Even such reports of outbreaks were attributed to low socioeconomic conditions similar to those observed in the developing nations [10-12].

\section{Indian scenario of diphtheria}

Recent reports of outbreaks and isolated case reports from developing countries like India reassert the fact that there is a possibility of the re-emergence of diphtheria in the post-vaccination era [13-15]. Also, reports of incidences of diphtheria from the combined state of Andhra Pradesh and the separated state of Telangana (India) support the fact that the infection is prevalent and that the pediatricians need to be cautious while diagnosing the suspected patients [16-17].

The epidemiological data of diphtheria in India appears to be inadequate. A recent article reported an analysis of diphtheria in India over the past two decades (1996-2016) [18]. This report suggested that diphtheria cases are frequent among school-going children and adolescents in India. The study had also noted that there was an $80 \%$ coverage of the initial three doses of vaccine and that there is no reliable data on the coverage of the booster dose. This report also observed that India accounts for more than half of the diphtheria cases reported worldwide (2001-2015). It also confirms that most states in India had reported outbreaks/cases of diphtheria, and amongst all, the combined Andhra Pradesh and Telangana reported an increased frequency of diphtheria cases (>1000 cases/year between 2005 and 2014) [18].

There have been several recent reports of outbreaks and newspaper articles highlighting the seriousness of the present situation, which emphasizes the role of the public, healthcare workers, and governments in order to control and prevent the spread of diphtheria [19-20]. A local newspaper published (in the Telegu language) a picture of parents and relatives carrying and transporting a pediatric patient to a better medical facility whose condition worsened with suspected diphtheria, as shown in Figure 7.

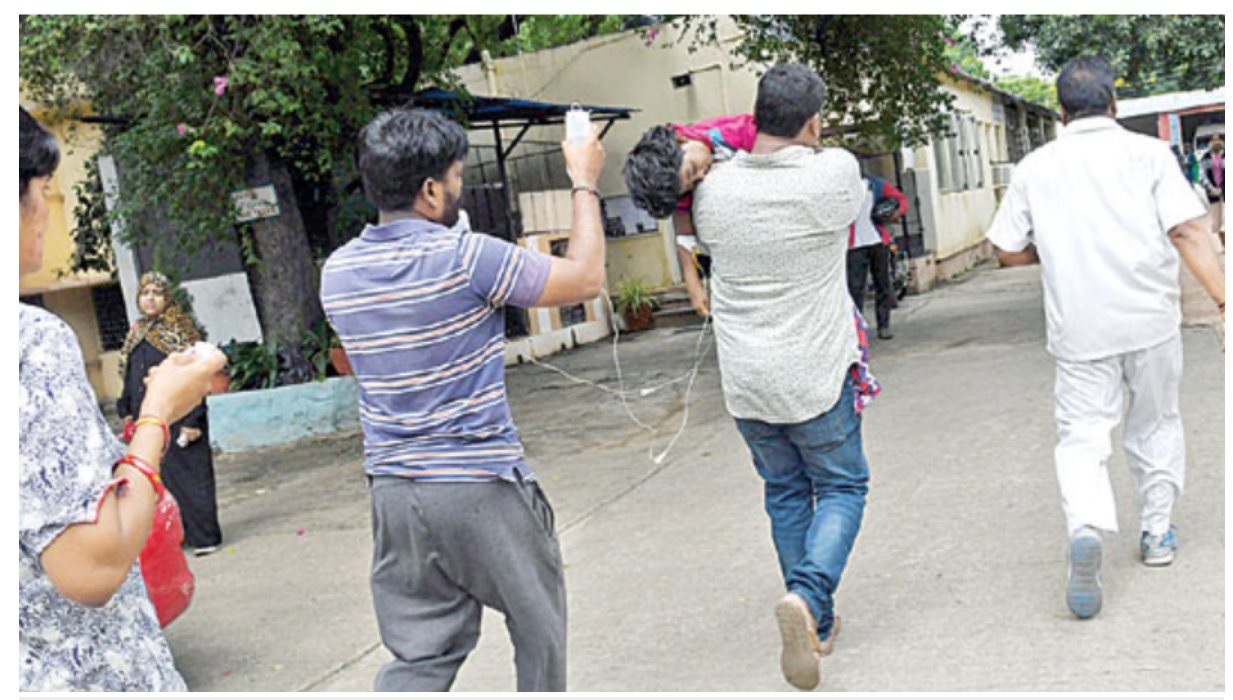

FIGURE 7: A child possibly suffering from diphtheria is being carried to a better medical facility by the relatives

Diphtheria has been controlled to a great extent with the introduction of DPT throughout the world. In spite of the vaccination, several studies in the past have reported the incidences of diphtheria globally. The 
condition in developing and financially constrained third-world countries appears to be worse due to illiteracy, malnutrition, overcrowding, and inadequate immunization. Isolated clinical cases and frequent reports of outbreaks of diphtheria-like infections should be adequately addressed in order to eliminate the infection. The governments should, therefore, actively perform surveillance of immunization as well as document the burden of morbidity and mortality associated with diphtheria.

\section{Conclusions}

The eight-year-old boy who presented with symptoms of fever, sore throat, and thickening of the posterior pharyngeal wall was provisionally diagnosed as a possible case of diphtheria. The laboratory confirmation of diphtheria was not possible because of inadequate facilities. The diagnosis was based on careful clinical and laboratory observations. The patient was isolated from others to avoid contact infections and was successfully treated with antibiotics and antidiphtheritic serum.

\section{Additional Information \\ Disclosures}

Human subjects: Consent was obtained by all participants in this study. Conflicts of interest: In compliance with the ICMJE uniform disclosure form, all authors declare the following: Payment/services info: All authors have declared that no financial support was received from any organization for the submitted work. Financial relationships: All authors have declared that they have no financial relationships at present or within the previous three years with any organizations that might have an interest in the submitted work. Other relationships: All authors have declared that there are no other relationships or activities that could appear to have influenced the submitted work.

\section{References}

1. Corynebacterium diphtheriae (diphtheria) . (2017). Accessed: November 7, 2019: http://www.antimicrobe.org/b99.asp.

2. Wolford RW, Schaefer TJ: Pharyngitis. Wolford RW, Schaefer TJ (ed): StatPearls Publishing, Treasure Island (FL); 2019.

3. Dandinarasaiah M, Vikram BK, Krishnamurthy N, Chetan AC, Jain A: Diphtheria re-emergence: problems faced by developing countries. Indian J Otolaryngol Head Neck Surg. 2013, 65:314-318. 10.1007/s12070-0120518-5

4. Diphtheria. A battle not yet won . (2017). Accessed: November 7, 2019: https://www.icmr.nic.in/sites/default/files/policy_brief/ICMR_NITM_ROY_Policy_Brief_Diptheria_Final_2017.pdf.

5. Review of the epidemiology of diphtheria - 2000-2016 . (2017). Accessed: November 7, 2019: https://www.who.int/immunization/sage/meetings/2017/april/1_Final_report_Clarke_april3.pdf.

6. Immunization, vaccines and biologicals. (2016). Accessed: November 7, 2019: http://www.who.int/immunization/monitoring_surveillance/data/en/.

7. Finger F, Funk S, White K, Siddiqui MR, Edmunds WJ, Kucharski AJ: Real-time analysis of the diphtheria outbreak in forcibly displaced Myanmar nationals in Bangladesh. BMC Med. 2019, 17:58. 10.1186/s12916019-1288-7

8. Dureab F, Al-Sakkaf M, Ismail O, Kuunibe N, Krisam J, Müller O, Jahn A: Diphtheria outbreak in Yemen: the impact of conflict on a fragile health system. Confl Health. 2019, 13:19. 10.1186/s13031-019-0204-2

9. Tosepu R, Gunawan J, Effendy DS, Ahmad LOAI, Farzan A: The outbreak of diphtheria in Indonesia. Pan Afr Med J. 2018, 31:249. 10.11604/pamj.2018.31.249.16629

10. Zalma VM, Older JJ, Brooks GF: The Austin, Texas, diphtheria outbreak: clinical and epidemiological aspects. JAMA. 1970, 211:2125-2129. 10.1001/jama.1970.03170130021004

11. Dixon JM: Diphtheria in North America. J Hyg (Lond). 1984, 93:2125-2129. 10.1017/s0022172400065013

12. Golaz A, Hardy IR, Strebel P, Bisgard KM, Vitek C, Popovic T, Wharton M: Epidemic diphtheria in the newly independent states of the former Soviet Union: implications for diphtheria control in the United States. J Infect Dis. 2000, 181:S237-243. 10.1086/315569

13. Krishnan S, Kizhakkekarammel P, George K, Johnson J, Kurukanari R, Raveendran G: Re-emergence of diphtheria in Malappuram district, North Kerala, India. J Acad Clin Microbiol. 2018, 20:37-39. 10.4103/jacm.jacm_2_18

14. Mohanty A, Bhatia M, Gupta P, Varshney S, Malhotra M, Omar BJ: Diphtheria: the patch still remains-a case report from the state of Uttarakhand. J Pharm Bioall Sci. 2019, 11:190-193. 10.4103/JPBS.JPBS_245_18

15. Nath B, Mahanta TG: Investigation of an outbreak of diphtheria in Borborooah block of Dibrugarh district, Assam. Indian J Community Med. 2010, 35:436-438. 10.4103/0970-0218.69282

16. Meera M, Rajarao M: Diphtheria in Andhra Pradesh-a clinical-epidemiological study . Int J Infect Dis. 2014, 19:74-78. 10.1016/j.ijid.2013.10.017

17. Asma, Ivaturi PB: A study on clinical and epidemiological profile of patients admitted with white patch in throat to Sir Ronald Ross Institute of Tropical and Communicable Diseases, Hyderabad, India. Int J Community Med Public Health. 2018, 5:1984-1989. 10.18203/2394-6040.ijcmph20181710

18. Murhekar M: Epidemiology of diphtheria in India, 1996-2016: implications for prevention and control . Am J Trop Med Hyg. 2017, 97:313-318. 10.4269/ajtmh.17-0047

19. Meshram RM, Patil A: Clinical profile and outcome of diphtheria in central India: a retrospective observational study. Int J Contemp Pediatr. 2018, 5:1600-1605. 10.18203/2349-3291.ijcp20182572

20. Rahman MR, Islam K: Massive diphtheria outbreak among Rohingya refugees: lessons learnt. J Travel Med. 2019, 26:tay122. 10.1093/jtm/tay122 